\title{
Una mirada a la crisis financiera internacional y sus relaciones con la disciplina contable y el ejercicio de la profesión: llamado a reflexionar sobre el deterioro de la imagen profesional*
}

\author{
Jorge Suárez Tirado**
}

Recibido: 15 de mayo de 2012

Aprobado: 23 de septiembre de 2012

\begin{abstract}
Suárez, J. (2012). Una mirada a la crisis financiera internacional y sus relaciones con la disciplina contable y el ejercicio de la profesión: llamado a reflexionar sobre el deterioro de la imagen profesional. Activos, 19, 21-37.
\end{abstract}

\section{JEL G10, Z00}

\section{Resumen}

Este artículo tiene como fin sistematizar ciertas apreciaciones con respecto a la reciente crisis financiera internacional (desde 2008 hasta el presente), describiendo algunas de sus causas y consecuencias, sin plantear recomendaciones para situaciones futuras. Se hace énfasis en las relaciones que se desarrollan entre la crisis financiera internacional y la disciplina y ejercicio de la profesión contable. Para ello, se realiza un estudio de literatura sobre los temas objeto de estudio, a partir de la revisión en revistas

\footnotetext{
*Artículo de reflexión no derivado de un trabajo investigativo (tipo X).

** Contador Público, magíster en Administración, docente tiempo completo de la Facultad de Contaduría Pública, Universidad Santo Tomás. Correo electrónico: jorgesuarez@usantotomas.edu.co
} 
y bases de datos de carácter nacional e internacional. Se culmina con algunos cuestionamientos, con reflexiones y con un llamado a repensar la disciplina contable, la falta de protección al interés público, las tendencias internacionales en contabilidad y la realidad como una construcción social, resultado de la acción humana.

Así pues, la crisis financiera internacional ha tenido impacto en los desarrollos contables y ha sido originada en parte por las trayectorias de la regulación contable internacional.

\title{
Palabras clave:
}

contabilidad, profesión contable, crisis financiera, interés público.

Suárez, J. (2012). A look at the international financial crisis and its relations with the accounting discipline and professional practice: a call to reflect on the deterioration of the professional image. Activos, 19, 21-37.

\begin{abstract}
This article aims to systematize certain appreciations with respect to the recent international financial crisis (from 2008 to the present), describing some of its causes and consequences, without making recommendations for future situations. Emphasis is placed on the relationships that develop between the international financial crisis and the discipline and exercise of the accounting profession. For this purpose, a study of literature on the topics under study is conducted, from the review in journals and databases, national and international. It ends with some questions, with reflections and with a call to rethink the accounting discipline, the lack of protection of the public interest, international trends in accounting and reality as a social construction, the result of human action.
\end{abstract}


Thus, the international financial crisis has had an impact on accounting developments and has been caused partly by the trajectories of international accounting regulation.

\section{Keywords:}

accounting, accounting profession, financial crisis, public interest.

\section{Suárez, J. (2012). Un regard de la crise financière internationale et ses relations avec la discipline comptable et l'exercice de la profession: appel à réfléchir sur la détérioration de l'image pro- fessionnelle. Activos, 19, 21-37.}

\section{Résumé}

Cet article a pour but de systématiser certaines appréciations par rapport à la récente crise financière internationale (de 2008 à nos jours), tout en décrivant certaines de ses causes et conséquences, sans faire des recommandations pour des situations futures. Le point est fait sur les relations qui se développent entre la crise financière internationale et la discipline et l'exercice de la profession comptable. Pour cela, une étude de littérature est faite sur les sujets objets d'étude, en partant de la révision de revues et des bases de données nationales et internationales. L’article finit avec quelques remises en question, avec des réflexions et avec un appel à repenser la discipline comptable, le manque de protection à l'intérêt publique, les tendances internationales en comptabilité et la réalité comme une construction sociale, résultante de laction humaine.

Ainsi, la crise financière internationale a eu un impact sur les développements comptables et a surgit en partie par les trajectoires de la régulation comptable internationale. 


\section{Mots-clés:}

comptabilité, profession comptable, crise financière, intérêt public.

Para llegar a la raíz de la crisis financiera, debemos reconocer una crisis cultural que no ha logrado ayudar a los empresarios ni a limitar el consumo y la producción ni a proporcionar una perspectiva moral y espiritual de la vida empresarial. Lo que muestra nuestra crisis financiera no son, en su mayor parte, personajes viciosos, sino personas con falta de carácter: una falta de consideración moral por parte de los agentes que persiguen ciegamente el dinero.

Naughton (2008, p. 34)

\section{Introducción}

Las crisis financieras son situaciones a las cuales se llega como resultado de problemas de liquidez que afectan el adecuado funcionamiento de las economías y los mercados. Este es el resultado de expansiones en los niveles de liquidez y el crédito bancario (García, 2008, p. 51). Tales crisis han tenido como antesala períodos sostenidos de crecimiento económico, en los cuales se desarrolla una política monetaria expansiva y, por ende, aumento de la liquidez y del crédito bancario. Estas medidas se presentan desde escenarios con excedentes de liquidez donde aumenta la demanda de créditos y hay alzas en el consumo de bienes y servicios; hecho que ocasiona subidas en los precios, lo cual trae como consecuencia mayor demanda de créditos.

Los altos niveles de liquidez, en especial en un país como Estados Unidos, son resultado de la captación incesante de recursos financieros, mediante la emisión de títulos del tesoro, provenientes de los superávits comerciales de algunos países como China e India (García, 2008, pp. 51, 52). 
El documento tiene como objetivo analizar ciertos rasgos propios de la crisis financiera que estalló en 2008, poniendo de manifiesto algunas de sus causas y consecuencias, seguido de un apartado más amplio en el cual se exponen las relaciones entre contabilidad, ejercicio profesional y el rol que ambas juegan en las crisis. Así pues, el documento se desarrolla a partir de la revisión de información bibliográfica proveniente de revistas y bases de datos nacionales e internacionales.

\section{Crisis económica y financiera}

Las crisis financieras se presentan cuando revienta una burbuja, la cual consiste en el aumento de precios de los activos, tanto reales como financieros, ocasionada por un exceso de demanda que se impulsa gracias al crédito bancario. La burbuja estalla cuando las expectativas sobre el aumento sostenido en los precios de los activos se estancan y se reversan. Esta situación origina una venta masiva de activos que provocan finalmente descenso en el precio de los mismos (García, 2008, pp. 50, 51).

En este contexto, es importante resaltar el alto nivel de desregulación de los sectores bancario y financiero desde los años ochenta del siglo XX, los cuales son propios del modelo económico neoliberal. En este orden, cabe destacar el efecto de la derogación en 1999 en Estados Unidos de la Ley Glass-Steagall (sancionada en 1933), que fue promulgada como resultado de la gran depresión y la cual buscaba separar la banca tradicional y de inversión, con el ánimo de proteger a los ahorradores de las inversiones riesgosas. Dicha ley se revocó gracias a la creencia neoliberal de la eficiencia en la autorregulación de los mercados financieros (Bouckaert, 2008, p. 11).

Así, mientras la banca tradicional o comercial se dirige al público con poco conocimiento sobre el funcionamiento de los mercados financieros y los riesgos, se hacen necesarios mayores niveles de regulación para proteger los recursos de los ahorradores; a su vez, la banca de inversión está dirigida hacia un público más especializado y con mayor grado de conocimiento sobre 
los riesgos en los que pueden incurrir al momento de optar por distintas alternativas de inversión. Debido al mayor nivel de conocimiento por parte de los agentes económicos, este sector presenta una regulación más flexible. El colapso en la banca de inversión se presenta por la forma tan compleja como funciona el mercado hipotecario y por la dinámica propia de la profunda globalización e integración del sistema financiero (García, 2008, p. 52).

\section{Causas de las crisis financieras}

Como algunas causas de las crisis financieras, se encuentra de manera general la ausencia de comportamientos éticos por parte de los agentes económicos, marcados por su avaricia y codicia; adicional a ello, los desmesurados procesos de liberalización y desregulación de los mercados, las inadecuadas políticas para valorar y gestionar los riesgos, el desarrollo del autointerés en las opciones de los inversionistas y la búsqueda del beneficio fácil y a corto plazo (Casilda, 2009, p. 93).

En particular, la crisis subprime de $2007^{3}$ tuvo origen en los altos niveles de préstamos orientados hacia la adquisición de vivienda, concedidos a familias que no ofrecían garantías; por lo tanto, eran irresponsables y de alto riesgo. Estos préstamos se agrupaban en paquetes de valores respaldados mediante activos que se vendieron en todo el mundo (Bouckaert, 2008, pp. 10, 11).

Para Larroulet (2008, p. 53), la crisis, más que ser una falla del mercado, es la manifestación más evidente de la crisis del Estado. Un ejemplo claro de esto es Estados Unidos, donde materializaron una política estatal para promover el endeudamiento de las familias orientado a la compra de vivienda, para sectores que no contaban con capacidad de pago para la

3. Abadía (2008) le ha dado a esta crisis la denominación de "ninja", ya que se aprobaban créditos a personas que no tenían ingresos ni trabajo y tampoco activos que sirvieran como respaldo ante los créditos. El término ninja implica entonces 'No Income, No Job, No Assets'. 
adquisición de la misma, medida que fue impulsada canalizando crédito a través de dos entidades intermediarias (Fannie Mae y Freddie Mac). Esto se acompañó de las medidas de expansión monetaria impulsadas por la Reserva Federal de los Estados Unidos (FED), que implicaban bajas tasas de interés para favorecer los niveles de endeudamiento.

Sin embargo, estas operaciones crediticias eran de alto riesgo debido a las bajas garantías que ofrecían los deudores y, cuando los mismos se materializaron, estalló la crisis de liquidez, financiera, de confianza y posteriormente económica.

Una característica relevante de la actual crisis financiera es que ha sido encubada por la financiarización de las economías occidentales, principalmente la economía de Estados Unidos que creó abundancia de créditos y animó la toma de riesgos mediante instrumentos financieros complejos y estructuras corporativas y mecanismos de regulación inefectivos (Ferguson, 2008; Morris, 2008; Soros, 2008). Los actores clave en este proceso fueron los bancos, fondos de cobertura y compañías de financiamiento (Sikka, 2009, pp. 868, 869).

Para Alford (2008, pp. 22-25), los sistemas de incentivos son precisamente un factor principal en las crisis, ya que estos resultan vitales como mecanismos para alinear los intereses de los agentes y evitar el autointerés. Asimismo, el optimismo excesivo, la falta de información, el crédito a bajo costo, los mercados globalizados y el no escuchar las advertencias son algunos de los motivos que llevaron a la crisis (García, 2008, p. 49). También se considera que la crisis financiera internacional se deriva de problemas de asimetrías de información, así como deficiente supervisión y regulación del sistema financiero (Katz, 2008, p. 56).

Por último, sin pretender agotar el listado de causas que llevaron a la crisis financiera subprime, hay que indicar las fallas en las tecnologías utilizadas para evaluar los riesgos asociados con los instrumentos financieros que llevaron a errores masivos en la valoración de activos; aspecto 
que trajo como resultado el quebrantamiento de la confianza crediticia. A pesar de que esta situación era conocida, no se hizo nada al respecto (Fontiveros, 2008, p. 77).

\section{Consecuencias de las crisis financieras}

Las crisis financieras implican una situación en la cual se presentan diversas distorsiones en las economías de los países. Dentro de esas distorsiones se pueden enumerar quiebras - Lehmann Brothers-, fusiones, compras, intervenciones estatales - AIG, Fannie Mae, Freddie Mac-, entre otras. Al respecto, Perry (2008, p. 63) afirmaba que: "Se ha requerido una inyección masiva de fondos públicos en todo el mundo industrializado para detener la hemorragia y aún no se sabe si el enfermo se salva”.

Cuando en las economías y los mercados hay problemas de liquidez, se restringe el crédito y con esto el consumo se ve afectado, se reducen los ingresos de las organizaciones, aumentan los despidos de trabajadores y disminuyen los recursos de los cuales disponen las familias para adquirir bienes y servicios. Esta es una de las manifestaciones del impacto que tienen las crisis financieras en la economía.

La reciente crisis también ha puesto en tela de juicio el gobierno corporativo de las organizaciones. Se han presentado debilidades en la composición de las juntas directivas - equilibrio entre miembros internos y externos-, en la calidad y efectividad de los mecanismos de control de gestión y rendición de cuentas, sumado a los paquetes de compensaciones para los directivos (Barrios, 2009, p. 8). Estos incentivos son creados para enfrentar los problemas de agencia que son riesgo moral y selección adversa. Igualmente, las crisis financieras se acompañan del quebrantamiento de la confianza existente en el estado, en la economía y en la capacidad del mercado para asignar eficientemente los recursos. Otro efecto negativo se encuentra en la desaceleración en los niveles de crecimiento económico (Katz, 2008, p. 56). 


\section{Contabilidad, ejercicio profesional y crisis financieras}

La contabilidad es una disciplina del conocimiento que busca representar la realidad económica de las organizaciones y, junto con la contaduría pública como ejercicio profesional, ha estado presente en las crisis financieras. La información contable permite la construcción de expectativas y con ello la toma de decisiones; por ende, bajo contextos de crisis, resulta prudente indagar con respecto a las implicaciones de disciplina contable y ejercicio profesional en este tipo de situaciones. A continuación se describen algunos elementos que ponen de manifiesto esta situación en la reciente crisis.

La contabilidad de la empresa es un recurso cognitivo de primer orden que brinda información con respecto a las actividades de las firmas y es fundamental para proveer información a los mercados financieros. Asimismo, puede entenderse como un instrumento para rendir cuentas registro y control de actividades realizadas - por parte de una organización, y que además participa en la creación del resultado, ya que este último está determinado por lo que la contabilidad representa (Aglietta y Rebérioux, 2009 , pp. 153, 154). Es una disciplina del conocimiento que tiene como funciones la localización de responsabilidades, evitar el fraude, orientar a la industria, definir capitales y determinar las utilidades (Hatfield, 1924, citado en Chatfield, 1979, p. 14). El interés exclusivo de la contabilidad es registrar hechos asociados a la propiedad y a los derechos de propiedad; las transacciones de esto último se reducen al dinero (Littleton, 1933, citado en Chatfield, 1979, pp. 25, 26).

Sumado a lo anterior, la contabilidad contribuye al aumento en el nivel de actividad económica cuando, al brindar información útil para la toma de decisiones, favorece la revelación en los niveles micro y macro; permite también aumentar la confianza entre ahorradores e inversionistas, hace posible el adecuado funcionamiento de los mercados de capitales, así como de las instituciones y entes económicos, hace posible el uso racional de los recursos de un país y estimula una correcta distribución de la renta y la riqueza (Tua, 2009, p. 4). 
Estas perspectivas permiten poner de manifiesto la importancia que tiene la contabilidad en el adecuado funcionamiento de las economías, las organizaciones y los mercados. De esta manera, se demanda por parte de los profesionales en materia contable la generación y divulgación de información que sea útil para apoyar los procesos de toma de decisiones de inversión y deuda principalmente.

Un aspecto que ha llevado a que la contabilidad esté relacionada con las crisis financieras es la ausencia del principio contable tradicional de prudencia, el cual busca que el ingreso y valor de la organización no sean sobreestimados (Maltby, 2000; Watts, 2003a, 2003b). De este modo, en la organización se impediría la distribución de ingresos y valorización de activos que no han sido realizados, es decir, se protegerían los recursos de los propietarios y otros grupos de interés.

Para Richard (2000) (citado en Aglietta y Rebérioux, 2009, p. 158), cuando se ignoran las plusvalías potenciales — valorización-, la empresa se evita tener que distribuir utilidades no realizadas entre sus accionistas; de este modo, se privilegia la continuidad en el proceso de explotación. Asimismo, el riesgo al que se exponen los administradores que marginan políticas conservadoras, con el fin de aumentar sus compensaciones, sería las posibles demandas que recibirían de los inversionistas por la pérdida en el valor de su inversión (Sunder, 2005, p. 224).

Teniendo en cuenta que Estados Unidos es un referente internacional en materia contable, autores como Zeff (2003a, p. 196) consideran que en este país, durante la década de los sesenta, la contabilidad, auditoría y profesión contable llegaron a su punto más alto en términos de reputación, pero después de este período esta imagen se ha deteriorado gradualmente. En los setenta se presenta un aumento en las litigaciones contra contadores y auditores, asociados con quiebras y escándalos corporativos, además de la pérdida de independencia de los auditores debido a que prestaban servicios de auditoría y consultoría a un mismo cliente. Con esto se generaba un conflicto de intereses y se ponía de manifiesto la ausencia de compromiso 
con la protección del interés público. Lo anterior se acompañó de un detrimento en los valores profesionales debido a que las presiones competitivas y la búsqueda en el crecimiento en las firmas de servicios contables profesionales estaban despertando actitudes comerciales en los $\mathrm{CPA}^{4}$, y se estaban dejando a un lado la cortesía, el respeto mutuo, el autocontrol y la justicia (Zeff, 2003b, p. 267).

Históricamente ha existido la concepción de que la contaduría pública salvaguarda la confianza pública y se encuentra alineada con el interés común; sin embargo, después de observar las crisis financieras y las quiebras empresariales, pareciera que ese objetivo no se ha cumplido y que, por el contrario, ha aumentado la brecha entre interés público y ejercicio profesional de la contaduría pública. La actual crisis que vive la profesión contable se presenta porque se han dejado a un lado los valores profesionales - objetividad, prudencia e independencia - en favor de la venta de servicios al mejor postor (Gómez, 2010). Asimismo, los colapsos corporativos inesperados, fraudes y fracasos aumentan los reclamos de experticia que se realizan a los auditores. Estas situaciones estimulan la sospecha de que los auditores faltan a los requisitos de independencia, experticia e incentivos para construir las prometidas cuentas verdaderas y razonables de los asuntos corporativos (Sikka, 2009, p. 868).

También se encuentra que el crecimiento de los servicios de consultoría en las ocho grandes firmas ${ }^{5}$ desde la mitad de los años setenta del siglo XX en adelante fue palpable, ya que los honorarios de consultoría como un porcentaje del total de los ingresos brutos de las empresas se incrementaron en un rango del 5 al $19 \%$ en 1977 y de 11 al 28 \% en 1984. Esto llevó a que en 1978 seis de las ocho grandes firmas estuvieran en el top 10 de las empresas de consultoría de los Estados Unidos en términos de facturación

4. Contadores públicos certificados (CPA, por sus siglas en inglés).

5. Actualmente debido a la reorganización del sector y por los efectos de la crisis, son solamente cuatro firmas. 
bruta por servicios de este tipo. Lo anterior se acompañó de crecimiento en el personal que laboraba en las firmas ya que, por ejemplo, Price Waterhouse (PW) pasó de 337 a 1300 empleados; para 1986, PW había evolucionado a tal punto que su misión fue convertirse nada menos que en una firma de asesoramiento de negocios de servicios integrales (Zeff, 2003b, p. 270).

Las firmas de auditoría ampliaron a nivel global sus mercados en los años cincuenta y sesenta, debido a la expansión en los negocios de sus clientes; la mayor parte de ellas concluyó aparentemente a inicios de los setenta que el mercado de la auditoría estaba fuertemente saturado y, por ende, compensaron esto con la agresiva ampliación de su escala y alcance en las prácticas de consultoría. La distribución de los mayores honorarios de las grandes firmas cambió considerablemente desde contabilidad y auditoría hacia impuestos y servicios de consultoría (Zeff, 2003b, pp. 270, 271).

En su trabajo que busca estimular el debate sobre las prácticas contemporáneas de la auditoría y la calidad de esta última al examinar informes financieros auditados de compañías en dificultades, Sikka (2009, p. 868) se pregunta por qué muchas empresas han estado buscando apoyo dentro de un breve período posterior a recibir opiniones de auditoría sin salvedades. Los auditores reciben grandes montos en honorarios de auditoría y consultoría; además, los eventos como la crisis financiera subprime aumentan las preguntas sobre los valores de las compañías de auditoría, la independencia del auditor, la calidad del trabajo de auditoría, incentivos económicos para buenas auditorías y el conocimiento básico de los auditores.

Si algo ha quedado claro con los recientes escándalos corporativos es que algunas prácticas con las cuales la contabilidad y los contadores han engañado al público consisten en acelerar el reconocimiento de ingresos, desacelerar el reconocimiento de gastos y mantener pasivos fuera del balance. Enron, una de las grandes empresas de los Estados Unidos por años, mantuvo deudas fuera del balance, aumentando los informes de ganancias; por su parte, WorldCom capitalizó gastos y Global Crossing realizó registros inadecuados de ingresos. El público aprendió que los contadores fallan al 
detectar esos problemas de contabilidad o ayudan a construir transacciones contables que no son cuestionadas (Young, 2005, p. 2).

Ante este tipo de situaciones, surgen algunos cuestionamientos. Por ejemplo, los creadores de política podrían tomar una pausa y preguntarse por qué muchas personas estuvieron voluntariamente involucradas en diversas actividades escandalosas; un elemento es la codicia, otro factor es el interés propio. Los ejecutivos buscaban más ingresos y beneficios y el aumento en el precio de las acciones; los auditores buscaban más honorarios y los analistas buscaban bonos más altos. La búsqueda por "más" impregna a la sociedad en todos los niveles: nacional — crecimiento de variables económicas - , organizacional -resultados de desempeñoe individual - progreso material- (Young, 2005, p. 3, 4).

Ante una crisis como la actual, emitir normas ${ }^{6}$ como solución a los graves problemas que afronta la contabilidad implica buscar respuestas esencialmente técnicas que intentan desanimar determinados comportamientos aunque estimulen otros; estas fueron diseñadas en un esfuerzo para reafirmar la confianza de los inversores en un funcionamiento razonable y eficiente de los mercados de capital. No se han cuestionado abiertamente, entre otros, la eficacia de un sistema de mercado, la utilidad de nuestras regulaciones e instituciones reguladoras, la responsabilidad de las corporaciones y la conveniencia de continuar la base de nuestra sociedad desde el propósito del "más".

Sumado a lo anterior, aunque las nuevas reglas son intentos para inducir el comportamiento ético y prevenir el delito o prácticas engañosas, la moralidad — vivir bien y hacer el bien — no es materia de seguir a ciegas reglas que existen o de encontrar las mejores reglas dentro de un sistema existente. La práctica moral requiere voluntad de cuestionar seriamente la continua aptitud de nuestros principios en uso, nuestros principios

6. Por ejemplo, Basilea III recientemente y la Ley Sarbanes Oxley de 2002, posterior a las quiebras de Enron y WorldCom. 
individuales, organizacionales e institucionales (Pappas, 1998; Rockefeller, 1991; Seigfried, 1996, citados en Young, 2005, pp. 9, 10).

Los informes financieros de buena calidad, que se espera sean producidos por los contadores públicos, no solamente dependen de contar con estándares de contabilidad de alta calidad. Esto se acompaña de incentivos de preparadores, auditores y usuarios de los informes financieros. Esos estímulos son influenciados a su vez por mecanismos de aplicación, infraestructura legal, estructura de gobierno, sistema de educación, formación, etc. (Jacob y Madu, 2009, p. 720).

\section{Consideraciones finales}

Tal como ha quedado de manifiesto, las crisis financieras han permeado a la disciplina contable y el ejercicio profesional, y han afectado así la imagen de las mismas debido a su presencia en las crisis financieras. Resulta pertinente acudir a Mattessich (2005, p. 13), quien considera que en la sociedad se requiere un cambio de la tendencia actual de avaricia desenfrenada y decadencia social; si esta situación no se presenta, los esfuerzos que algunos contables y funcionarios realicen para mejorar la moralidad no serán suficientes.

Del mismo modo, desde las actuales tendencias internacionales en materia contable, se está rompiendo con la contabilidad como un instrumento para rendir cuentas, ya que se deja de informar sobre los actos de las empresas para hacer énfasis en aquello que, según el mercado, la empresa hará (Aglietta y Rebérioux, 2009, pp. 163, 164). Esto demanda una revisión cuidadosa y diligente de los cambios que las nuevas tendencias internacionales en materia contable presentan sobre las bases conceptuales e instrumentales de la contabilidad.

La reputación de la profesión contable se encuentra en duda, ya que los profesionales en este campo no están contribuyendo a la protección del 
interés público. Por eso, en el actual estado del mundo, tal como lo plantea Gómez (2010), resulta fundamental comprender la noción de interés público subyacente a los principios, estructuras, regulaciones y acciones de los contadores públicos en los últimos tiempos, con el fin de adquirir herramientas que permitan generar aportes para clarificar, reinterpretar y reposicionar las relaciones entre contaduría pública y bien común, para enfrentar los retos de la sociedad. Además, si bien los referentes internacionales pueden tener sus bondades en términos de relaciones humanas, sociales y económicas, aun así deben ser objeto de análisis, con el fin de evaluar de manera crítica sus posibilidades y limitaciones. De allí que resulte vital la revisión de trabajos como los de Demski, Fellingham, Jjiri y Sunder (2002), donde los autores exponen algunas reflexiones propias de los fundamentos intelectuales de la contabilidad, así como las reflexiones planteadas por González (2010) sobre la enseñanza instrumental de la profesión contable.

Por último, no se puede olvidar que el orden social es una construcción humana que no se da biológicamente, no es parte de la naturaleza de las cosas y no puede derivarse de leyes naturales. De esta manera, el orden social solamente existe como producto de la acción humana (Luckmann y Berger, 2008, p. 71) y situaciones como las crisis financieras y la presencia en ellas de los profesionales en materia contable no son ajenas a este escenario, y deben ser pensadas como un producto social resultado del comportamiento de los seres humanos.

\section{Referencias}

Abadía, L. (2008). La crisis financiera NINJA. Recuperado de http://leopoldoabadia. blogspot.com/search/label/\%2B\%201\%20La\%20Crisis\%20NINJA

Aglietta, M. y Rebérioux, A. (2009). El debate contable. En El capitalismo financiero a la deriva: el debate sobre el gobierno de empresa (pp. 151-182). Bogotá: Universidad Externado de Colombia.

Alford, H. (2008, diciembre). La crisis y los sistemas de incentivos. Revista Cultura Económica, XXVII(73), 22-25. 
Bouckaert, L. (2008, diciembre). La catarsis financiera. Revista Cultura Económica, XXVI(73), 10-13.

Casilda, R. (2009). Las navajas sistémicas de la crisis financiera mundial: estado, mercados y bancos. Revista de Economía Mundial, 23, 87-106.

Chatfield, M. (1979). Estudios contemporáneos en la evolución del pensamiento contable. Belmont: Dickenson Publishing Company, Inc.

Demski, J., Fellingham, J., Ijiri, Y. y Sunder, S. (2002, junio). Some thoughts on the intellectual foundations of accounting. Accounting Horizons, 16(2), 157-168.

Fontiveros, D. (2008). Venezuela y la crisis del capitalismo mundial. Revista Latinoamericana de Política, Economía y Sociedad Perspectiva, 19, 76-78.

García, G. (2008). La crisis financiera: lo nuevo, lo viejo y sus implicaciones. Debates IESA, XIII(4), 50-53.

Gómez, M. (2010). Interés público y ejercicio de la contaduría pública: miradas al contexto internacional y aprendizaje para la profesión en Colombia. Recuperado de http://asis.umariana.edu.co/publicaciones_unimar/index. php?option=com_contentyview $=$ articleyid=306:revista-unimar-no-54-interes-publico-y-ejercicio-de-la-contaduria-publica-miradas-al-contexto-internacional-y-aprendizaje-para-la-profesion-en-colombiaycatid=61yItemid=196

González, J. (2010, enero-junio). Desprofesionalización de la enseñanza y desarrollo del saber contable. Revista Activos, 14, 19-27.

Hatfield, H. (1979). Defensa histórica de la contabilidad. En M. Chatfield (Comp.), Estudios contemporáneos sobre la evolución del pensamiento contable (pp. 1-14). México: Editorial ECASA.

Jacob, R. y Madu, C. (2009). International financial reporting standards: an indicator of high quality. International Journal of Quality y Reliability Management, 26(7), 712-722.

Katz, I. (2008). México y el capitalismo. Revista Latinoamericana de Política, Economía y Sociedad Perspectiva, 19, 56-58.

Larroulet, C. (2008). Lecciones para enfrentar la crisis: el caso de Chile. Revista Latinoamericana de Política. Economía y Sociedad Perspectiva, 19, 52-54. Littleton, A. (1979). Los antecedentes de la contabilidad por partida doble. En M. Chatfield (Comp.), Estudios contemporáneos en la evolución del pensamiento contable (pp. 25-34). México: Editorial ECASA. 
Luckmann, T. y Berger, P. (2008). La construcción social de la realidad. Buenos Aire: Amorrortu.

Maltby, J. (2000). The origins of prudence in accounting. Critical Perspectives on Accounting, 11, 51-70.

Mattessich, R. (2005). Lecciones de Enron y Arthur Andersen Co. [Ensayo publicado en línea]. Recuperado de http://www.mgeu.uma.es/CONFERENCIAS/ Enronmalaga_LV.pdf

Naughton, M. (diciembre, 2008-mayo, 2009). Una gran oportunidad para una reforma moral. Revista Cultura Económica, XXVI(73)/XXVII(74), 34-37.

Perry, G. (2008). Lecciones de la crisis financiera. Revista Latinoamericana de política, Economía y Sociedad Perspectiva, 19, 62-65.

Sikka, P. (2009). Financial crises and the silence of the auditors. Accounting Organizations and Society 34, 868-873.

Sunder, S. (2005). Teoría de la contabilidad y el control. Bogotá: Universidad Nacional de Colombia.

Tua, J. (2009). Contabilidad y desarrollo económico. El papel de los modelos contables de predicción. Especial referencia a las Nic's. Recuperado de http:// www.elcriterio.com/revista/ajoica/contenidos_4/contabilidad_y_desarrollo_cali_09.pdf

Watts, R. (2003a, diciembre). Conservatism in accounting part I: explanations and implications. Accounting Horizons, 17(3), 207-221.

Watts, R. (2003b, diciembre). Conservatism in accounting part II: evidence and research opportunities. Accounting Horizons, 17(4), 287-301.

Young, J. (2005). Changing our questions: reflections on the corporate scandals. Accounting and the Public Interest, 5, 1-14.

Zeff, S. (2003a septiembre). How the U.S. accounting profession got where it is today: part I. Accounting Horizons 17(3), 189-205.

Zeff, S. (2003b, diciembre). How the U.S. accounting profession got where it is today: part II. Accounting Horizons, 17(4), 267-286. 